\title{
Bee- and Wasp-Venom Sensitization in Schoolchildren of High- and Low-Socioeconomic Status Living in an Urban Area of Indonesia
}

\author{
Aldian I. Amaruddin ${ }^{a, b}$ Jan Pieter R. Koopman ${ }^{b}$ Munawir Muhammad ${ }^{\text {, d }}$

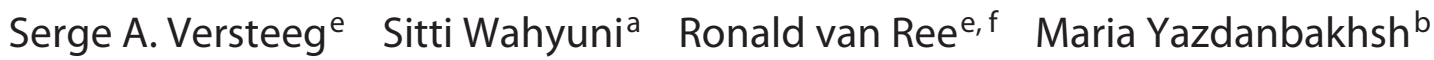 \\ Firdaus Hamid $^{c}$ Erliyani Sartono ${ }^{b}$ \\ aDepartment of Parasitology, Faculty of Medicine, Hasanuddin University, Makassar, Indonesia; ${ }^{b}$ Department of \\ Parasitology, Leiden University Medical Center, Leiden, The Netherlands; ' ${ }^{\circ}$ epartment of Microbiology, Faculty \\ of Medicine, Hasanuddin University, Makassar, Indonesia; 'Department of Microbiology, Faculty of Medicine, \\ University of Muhammadiyah Makassar, Makassar, Indonesia; ${ }^{\circ}$ Departments of Experimental Immunology, \\ Amsterdam UMC Location AMC, Amsterdam, The Netherlands; 'Departments of Otorhinolaryngology, Amsterdam \\ UMC location AMC, Amsterdam, The Netherlands
}

\section{Keywords}

Venom and insect allergy · Epidemiology · Pediatrics ·

Urban · Developing country

\begin{abstract}
Background: There is not much known about venom allergy in tropical regions. Here, we studied the prevalence of specific $\lg \mathrm{E}$ (slgE) and skin prick test (SPT) reactivity and reported sting-related symptoms, in high- and low-socioeconomic status (SES) schoolchildren living in urban city of Makassar in Indonesia. Methods: Children from high- $(n=160)$ and low( $n=165)$ SES schools were recruited. Standardized questionnaires were used to record information on allergic disorders as well as sting-related symptoms. Parasitic infection, SPT reactivity, and slgE to Apis mellifera (bee-venom) as well as Vespula spp. (wasp-venom) were assessed. Results: SPT reactivity to bee- and wasp-venom was 14.3 and $12.7 \%$, while the prevalence of slgE was 26.5 and $28.5 \%$, respectively. When SES was considered, prevalence of SPT to bee- and wasp-
\end{abstract}

venom was higher in high-SES than in low-SES schoolchildren (bee: 22.8 vs. $5.7 \%, p<0.001$; and wasp: 19.6 vs. $5.7 \%, p$ $<0.001)$. Conversely, slgE to both venoms was lower in highSES than in low-SES (bee: 19 vs. 34\%, $p=0.016$; and wasp: 19 vs. $38 \%, p=0.003$ ). Furthermore, among SPT positive subjects, considerable proportion had no detectable slgE to bee- $(65.85 \%)$ or wasp-venom (66.67\%). Altogether the sensitizations were rarely translated into clinical reaction, as only 1 child reported significant local reaction after being stung. No association with parasitic infections was found. Conclusions and Clinical Relevance: Sensitization against bee- or wasp-venom is quite prevalent among schoolchildren in Indonesia. The discordance between SPT and slgE might suggest the direct (non-lgE) effect of venoms in skin reactivity. Recorded sensitizations had poor clinical relevance as they rarely translated into clinical symptoms.

(c) 2021 The Author(s)

Published by S. Karger AG, Basel

Edited by: H.-U. Simon, Bern. karger@karger.com www.karger.com/iaa

Karger $\stackrel{\text { ' }}{=}$

BOPEN ACCESS
(C) 2021 The Author(s)

Published by S. Karger AG, Basel

This is an Open Access article licensed under the Creative Commons Attribution-NonCommercial-4.0 International License (CC BY-NC) (http://www.karger.com/Services/OpenAccessLicense), applicable to the online version of the article only. Usage and distribution for commercial purposes requires written permission.
Correspondence to:

Firdaus Hamid, firdaus.hamid@gmail.com

Erliyani Sartono, e.sartono@lumc.nl 


\section{Introduction}

The global magnitude of venom allergy is not completely established as the majority of studies have been conducted in temperate or subtropical countries. The prevalence of Hymenoptera venom sensitization has been reported to vary from 3.66 to $41.6 \%$ [1-5], whereas prevalence of systemic allergic reactions to venoms has been estimated to be between 0.34 and $16 \%$ [3, 6-9]. A single Hymenoptera sting may induce large local reactions (LLRs) and/or systemic reactions (SRs) in venomallergic individuals, and in some instances, SR can be fatal [10]. Altogether, there is not much information on the extent of venom allergy and symptoms in tropical countries.

Indonesia, a tropical country, located across the equator with a warm climate and high humidity, provides favorable conditions for Hymenopterans like bees and wasps. To gather information on venom allergy in Indonesia, we conducted a study among schoolchildren of high- and low-socioeconomic status (SES) living in Makassar, Sulawesi. We investigated the prevalence of reported Hymenoptera sting reactions and their correlation with Hymenoptera venom-specific IgE (sIgE) and skin reactivity. As the prevalence of parasitic infections can be high in children of low SES [11] and inverse relationship between aeroallergens and helminth infections have been reported [12], we also assessed whether the same is true for venom allergy.

\section{Material and Methods}

\section{Study Area and Design}

This study was approved by Health Research Ethics Committee of Faculty of Medicine, Hasanuddin University (Ref: 1504/ H04.8.4.5.31/PP36-KOMETIK/2016). Of 500 children (low SES: $n=250$; high SES: $n=250)$ invited, 325 children (65\%) participated in the study.

The study was conducted in 2 elementary schools in Makassar, Indonesia. Two elementary schools that were distinct in SES were selected. The low-SES school was located near the seaport where majority of parents earned wages by working in fishing or other low-education labor. Students from this school mainly lived in the school neighborhood in a densely populated area which is located near a landfill. The high-SES school was situated in the city center, and all students were living scattered over the city. The majority of the students' parents worked as moderate-to-high level civil servants or as professionals in business sector.

After discussion with the school management team and obtaining their agreement to participate in the study, a letter with detailed information on the study was sent to parents of all children in 3rd and 5th grade in both high- and low-SES schools. The parents could call the study team with any questions regarding the study.
We asked the parents' permission to include their children in the study, which was indicated by signing the informed consent letter. Only children who returned the signed letters were enrolled in the study.

\section{Questionnaires}

Standardized questionnaire to gather data regarding demographic and SES [11] as well as information on Hymenoptera sting allergy [13] was administered to the parents. In addition, questions about self-reported asthma, rhinitis, and eczema were asked using a questionnaire which was modified from ISAAC questionnaires when translated into Bahasa Indonesia (see online suppl. questionnaires; see www.karger.com/doi/10.1159/000516155for all online suppl. material).

The parents were asked whether the children ever helped their parents with beekeeping or gardening, and some sting-related questions such as, whether their children had ever been stung by bee or wasp, and whether they noticed any response after the sting such as a LLR, characterized by a swelling defined to exceed $10 \mathrm{~cm}$ that lasts for $>24 \mathrm{~h}$, and a SR, characterized as generalized hives or angioedema after the sting, or breathing difficulties, or loss of consciousness after the sting [14]. The questionnaires were all translated into Bahasa Indonesia and answered as a Yes or No.

\section{Skin Prick Testing}

SPT reactivity to bee-venom (Apis mellifera) and wasp-venom (Vespula spp.) was tested using allergen extract at concentrations $300 \mu \mathrm{g} / \mathrm{mL}$ for each venom (Soluprick-SQ, ALK-Abello, Madrid, Spain). SPT reactivity to mould (Aspergillus spp.), cockroach (Blattella germanica), and house dust mites (HDMs) such as Dermatophagoides pteronyssinus and Dermatophagoides farinae were also tested (Soluprick-SQ, ALK-Abello, Madrid, Spain). Histamine dihydrochloride $(10 \mathrm{mg} / \mathrm{mL})$ was used as positive control, meanwhile, allergen diluents as the negative control (Soluprick-SQ, ALK-Abello, Madrid, Spain). This measurement was performed using standard protocol as described previously $[11,15]$. SPT was carried out by 1 trained research staff and measured by another staff for whole study population. Wheal size was assessed at 15 min by measuring mean of perpendicular diameter. Wheal size $\geq 3 \mathrm{~mm}$ was considered as positive. Anthropometric measurements such as body weight and height were also performed.

\section{Specific and Total IgE Measurements}

Based on availability of sufficient serum samples and considering budgetary restriction, all 70 samples of subjects who were SPT positive to any venom and 130 samples of randomly selected subjects negative for SPT to any venom were selected for measurements of sIgE against D. pteronyssinus, bee-, and wasp-venom.

Total IgE levels in serum were measured using ELISA as previously described with minor modification [16]. For this assay, serum sample was diluted 50 times using PBS containing $0.05 \%$ Tween-20. The results were expressed as IU/mL. Allergen sIgE antibodies against HDM D. pteronyssinus (d1), bee-, (i1) and waspvenom (i3) from serum samples were determined by the ImmunoCAP system according to the manufacturer recommendation (Thermo-Fisher Scientific, Uppsala, Sweden). Specific IgE levels $\geq 0.35 \mathrm{kU} / \mathrm{L}$ were interpreted as positive. 


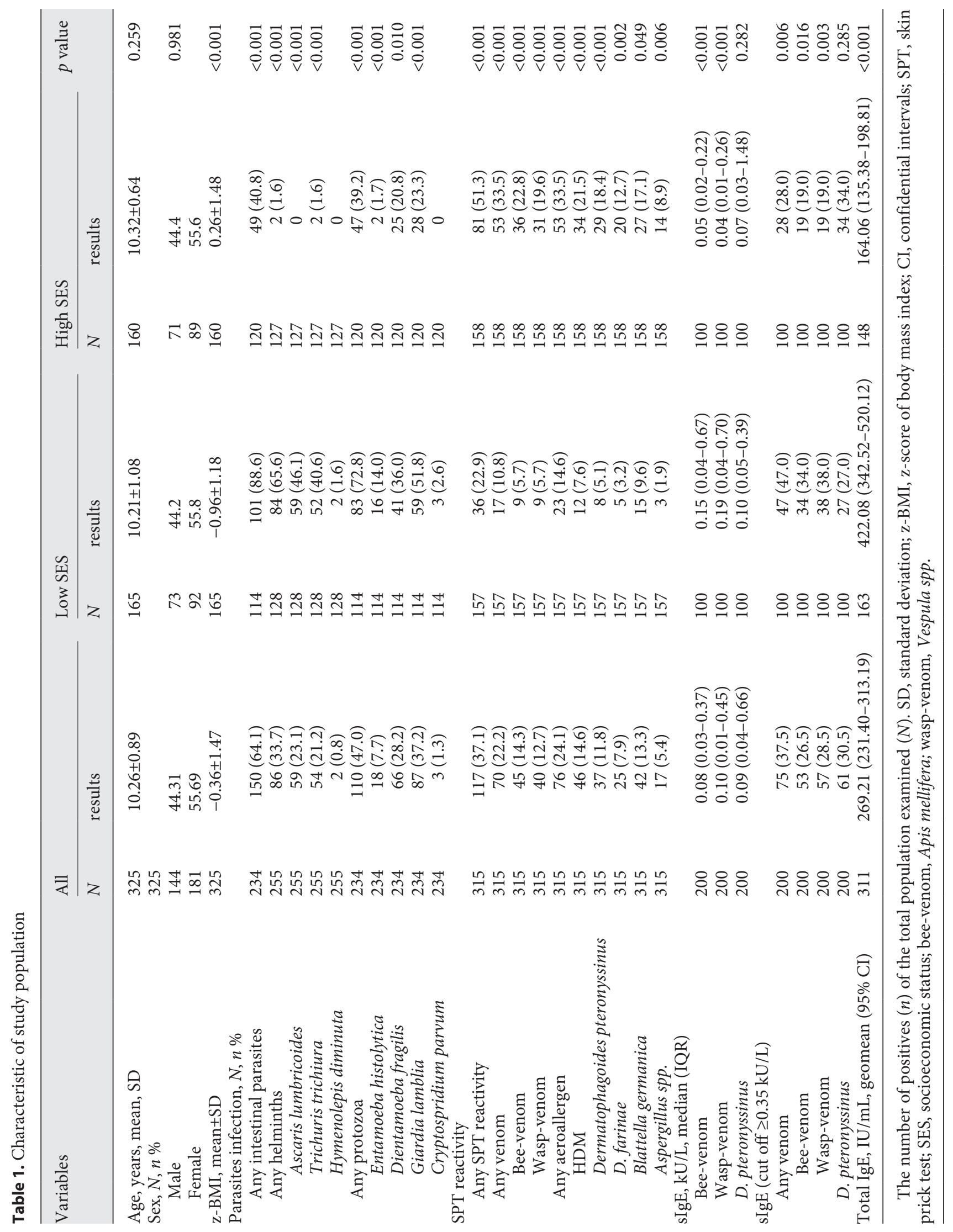


Parasitological Examination

Stool samples collected from children were used to assess intestinal helminths and protozoa infection. Kato-Katz method on stool samples was performed to quantify eggs from soil-transmitted helminths such as Ascaris lumbricoides and Trichuris trichiura. Aliquots of fresh stool samples were preserved in $-80^{\circ} \mathrm{C}$ for further analysis. DNA extraction was performed from frozen stool sample as described elsewhere [17]. PCR was used for detection of Entamoeba histolytica, Dientamoeba fragilis, Giardia lamblia, and Cryptosporidium spp. as has been described in detail previously [18-20].

\section{Statistical Analysis}

Central distribution of continuous variables was presented as mean \pm SD if the data were normally distributed and as median (IQR) if not normally distributed. Total IgE level was log-transformed, and the value was presented as geometric mean $(95 \% \mathrm{CI})$. Categorical variables were presented as proportion. Differences between 2 groups were tested using Student's $t$ test or Mann-Whitney $U$ for continuous data or $\chi^{2}$ for categorical data. Logistic regression was used to analyse potential factors that might contribute to the development of sensitization to allergens tested. In multivariate analysis, we included age, sex, zBMI as a priori confounders, as well as other variables that were significant in univariate analysis.

The agreement between skin test and IgE positivity was analyzed using Cohen Kappa test, while the Spearman rank correlation was used to assess the correlation between skin wheal size and the levels of sIgE [21]. Agreement is defined as having SPT positive with corresponding sIgE positive or having SPT negative with corresponding sIgE negative.

All statistical measurements considered to be significant if $p$ value $<0.05$. Statistical analysis was performed using IBM Statistical Package for the Social Science Statistics version 25 (IBM Corp., Armonk, NY, USA).

\section{Results}

\section{Characteristics of the Study Populations}

In total, 325 children were recruited from low- $(n=$ $165)$ and high- $(n=160)$ SES schools. No differences in terms of age and sex were observed between the 2 groups (Table 1). Low-SES children had significantly lower $\mathrm{z}$ BMI than children from high-SES school (mean \pm SD: $-0.96 \pm 1.18$ vs. $0.26 \pm 1.48$; respectively; $p$ value $<0.001$ ). Stool samples were collected from 255 (low SES, $n=128$; high SES, $n=127$ ) children. There was no difference between children who provided stool samples and those who did not in terms of age, sex, and zBMI. Helminths (65.6 vs. $1.6 \%$; $p$ value $<0.001)$ and protozoa infections ( 72.8 vs. $39.2 \%$; $p$ value $<0.001$ ) were more prevalent in low- than high-SES schoolchildren (Table 1). The levels of total IgE were higher in low- than in the high-SES schoolchildren (geomean [95\% CI]: 422.08 [342.52520.12 ] vs. 164.06 [135.38-198.81]; $p$ value $<0.001$ ).

Bee- and Wasp-Venom Sensitization in Urban Area of Indonesia

\section{Sensitization to Aeroallergens and Venoms}

SPTs were performed in 322 children (low SES $=164$ and high SES =158). After excluding 7 children of the low SES group with a histamine negative response, we found no differences in histamine wheal size between low- $(n=$ $157)$ and high- $(n=158)$ SES schoolchildren (mean \pm SD, $6.1 \pm 0.9 \mathrm{~mm}$ vs. $6.3 \pm 0.9 \mathrm{~mm}$, respectively). Next, when we compared the wheal size of SPT to each $D$. pteronyssinus, D. farinae, cockroach, mould, bee- and wasp-venom allergen, between low and high SES and observed no differences in wheal size of skin response to allergens tested, except for bee venom which is slightly larger in the low SES than in the high SES (median [IQR]: $4.0 \mathrm{~mm}$ [3.25$4.25]$ and $3.0 \mathrm{~mm}$ [3.0-3.5], respectively) (online suppl. Table 1).

The highest percentage of positive SPT was to bee-venom (14.3\%), followed by cockroach (13.3\%), wasp-venom (12.7\%), D. pteronyssinus (11.8\%), D. farinae (7.9\%) and mould (5.4\%). Strikingly, for all allergens tested the percentage positive SPT was significantly higher in highthan low-SES schoolchildren. Positivity for any SPT was 51.3 versus $22.9 \%$, in high versus low-SES group ( $p$ value $<0.001)$ and for specific allergens the values were 22.8 versus $5.7 \%$ to bee-venom ( $p$ value $<0.001$ ); 17.1 versus $9.6 \%$ to cockroach ( $p$ value $=0.049) ; 19.6$ versus $5.7 \%$ to waspvenom ( $p$ value $<0.001) ; 18.4$ versus $5.1 \%$ to $D$. pteronyssinus ( $p$ value $<0.001) ; 12.7$ versus $3.2 \%$ to $D$. farinae ( $p$ value $=0.002)$; and 8.9 versus $1.9 \%$ to mould ( $p$ value $=$ 0.006 ).

In the low-SES school, there was no difference in zBMI of children who were positive or negative for allergen SPT (online suppl. Table 2). Moreover, no differences were found in the prevalence of parasitic infection between subjects positive and negative skin test reactivity (online suppl. Table 3).

In contrast to skin test reactivity, analysis of allergen sIgE revealed that the prevalence of IgE positivity to both venoms was higher in low- than high-SES school (34.0 vs. $19.0 \%, p$ value $=0.016$, for bee-venom; and 38 vs. $19 \%, p$ value $=0.003$, for wasp-venom). The percentage of children with IgE positivity to both bee- and wasp-venom was $17.5 \%$ (25\% in the low SES and $10 \%$ in the high SES, $p$ value $=0.005)$. There were no differences in the $\operatorname{sigE}$ positivity to $D$. pteronyssinus between the 2 groups (Table 1).

\section{Potential Factors Associated with sIgE Positivity and SPT Reactivity to any Venom}

In univariate analysis, skin reactivity was positively associated with high SES (OR, 4.52; 95\% CI, 2.28-7.59) 
Table 2. Proportion of tests with discordant results between sIgE and skin reactivity to D. pteronyssinus, bee- and wasp-venom in low- and high-SES schoolchildren

\begin{tabular}{lccc}
\hline & D. pteronyssinus & Bee-venom & Wasp-venom \\
\hline a Proportion of sIgE negative among SPT positive schoolchildren & & \\
All, $n / N(\%)$ & $5 / 30(16.7)$ & $27 / 41(65.9)$ & $26 / 39(66.7)$ \\
Low-SES, $n / N(\%)$ & $1 / 7(14.3)$ & $4 / 9(44.4)$ & $2 / 9(22.2)$ \\
High-SES, $n / N(\%)$ & $4 / 23(17.4)$ & $23 / 32(71.9)$ & $24 / 30(80.0)$ \\
\hline b Proportion of SPT negative among sIgE positive schoolchildren & & \\
All, $n / N(\%)$ & $36 / 61(59.0)$ & $37 / 51(72.6)$ & $44 / 57(77.2)$ \\
$\quad$ Low-SES, $n / N(\%)$ & $21 / 27(77.8)$ & $27 / 32(84.4)$ & $31 / 38(81.6)$ \\
High-SES, $n / N(\%)$ & $15 / 34(44.1)$ & $10 / 19(52.6)$ & $13 / 19(68.4)$ \\
\hline
\end{tabular}

Data presented as (a) percentage of sIgE negative $(n)$ among sIgE positive schoolchildren $(N)$ and (b) percentage of SPT negative $(n)$ among sIgE positive $(N)$ schoolchildren. SPT, skin prick test; SES, socioeconomic status; Vespula spp.

while negatively associated with the presence of helminth infection (OR, 0.26; 95\% CI, 0.12-0.59). No association with zBMI was observed. In contrast to SPT positivity, sIgE was negatively associated with high SES and high zBMI (OR, 0.45; 95\% CI, 0.25-0.81 and OR, 0.77; 95\% CI, 0.62-0.96; respectively). No association was observed between sIgE positivity and current helminth infection.

Multivariate analysis adjusted with age, sex, and zBMI revealed that skin reactivity to any venom remained associated with high SES (OR [95\% CI], adjusted $p$ value: 5.15 [2.66-9.97]; p.adj <0.001). Similarly, following adjustment with age, sex, and zBMI, the negative association between high SES and sIgE remained intact $(0.52$ [0.27-0.98]; p.adj $=0.042$ ).

\section{Reported Clinical Symptoms}

The sting-related questionnaire was assessed in 151 children with completed data on SPT and sIgE to any venom. Of these children, 17 (11.3\%) had been stung by Hymenopterans at least once in their lifetime and experienced a local reaction such as sharp burning pain, redness, and slight swelling at the location after being stung and among them, 1 (5.9\%) child reported to have a history of LLR, while none did report SR after being stung. The child, who has reported to have LLR, had a positive skin test but negative sIgE to any venom. Among the remaining 16 children who had been stung but had no reported LLR, 7 were positive for both SPT and sIgE to any venom, while 9 children had positive SPT but were negative for sIgE to any venom.

Next, we analyzed the association between sIgE and skin sensitization to $D$. pteronyssinus and self-reported clinical symptoms of allergy among 151 subjects who had completed data. The Venn diagram in online suppl. Figure 1 shows the overlap between sensitization and selfreported and/or ever-diagnosed allergic asthma, rhinitis, or eczema. Among 22 children who reported clinical symptoms of allergy, $16(72 \%)$ were positive in sIgE to $D$. pteronyssinus, of which, 10 children were also positive for SPT against $D$. pteronyssinus.

\section{Discordance between Specific IgE and Skin Test Reactivity}

To assess discordance between SPT and sIgE, we selected individuals with positive SPT among 197 subjects, for whom we had both SPT and IgE data. When analyzing 41 subjects with positive SPT to bee-venom, we found 27 (65.9\%) were sIgE negative. Similarly, among 39 subjects that were SPT positive to wasp-venom, $26(66.7 \%)$ were sIgE negative. However, of 30 children with positive SPT to $D$. pteronyssinus, there were only 5 children (16.7\%) that were negative for sIgE to D. pteronyssinus (Table 2A; online suppl. Fig. 2).

Furthermore, the proportion of SPT negative among sIgE positive to bee- and wasp-venom was 72.6 and $77.2 \%$, respectively. When we stratified the analysis based on SES, the proportion of discordance was higher in the low SES than the high SES and was statistically significant for bee-venom (bee-venom: 84.4 and 52.6\%, $p=0.01$; waspvenom: 81.6 and $68.4 \%, p=0.27$, respectively). The proportion of SPT negatives among sIgE positive to $D$. pteronyssinus was also assessed. Among 61 children with positive sIgE, 36 children (59.0\%) were negative for SPT; with higher discordance in the low SES (77.8\%) than the high SES (44.1\%) (Table 2B; online suppl. Fig. 2).
Amaruddin et al. 


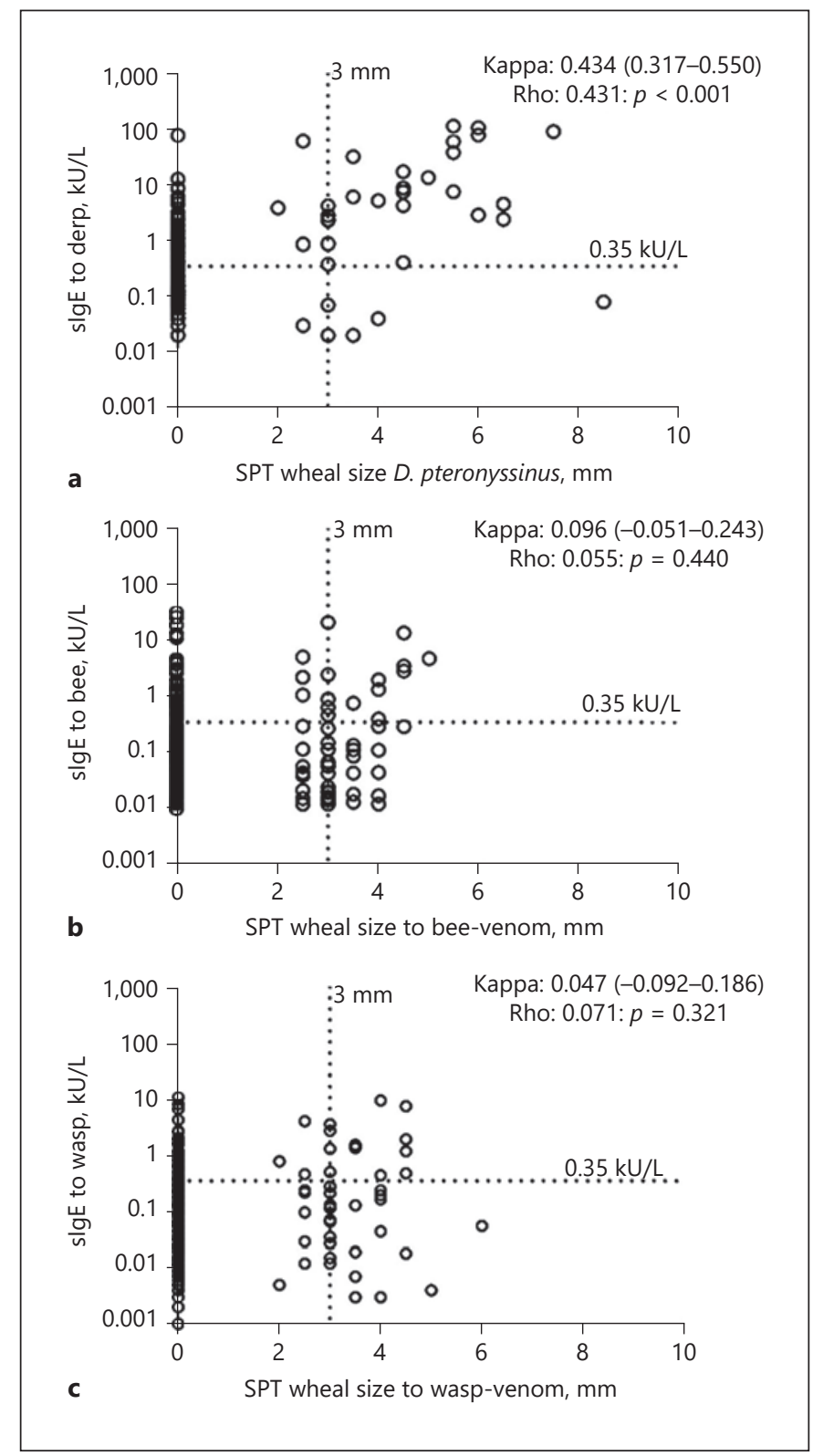

Fig. 1. Comparison between SPT wheal size and SIgE to D. pteronyssinus (a); bee venom (b); and wasp venom (c). Dotted lines show sensitization cutoff of $0.35 \mathrm{kU} / \mathrm{L}$ for sIgE and $3 \mathrm{~mm}$ for SPT wheal size. Agreement between SPT positivity and sIgE positivity was tested using Kappa Cohen's test and presented as Kappa (95\% CI). Correlation between SPT wheal size and level of sIgE to allergen tested presented as Spearman correlation coefficients (rho) and $p$ values. SPT, skin prick test; bee-venom, Apis mellifera; waspvenom, Vespula spp.

In Figure 1, we plotted the wheal size of skin reactivity against the sIgE levels. The results showed a "none to slight" agreement for bee- or wasp-venom sensitization $($ Kappa $=0.096[-0.051-0.243], p$ value $=0.175$, Fig. $1 \mathrm{~b}$

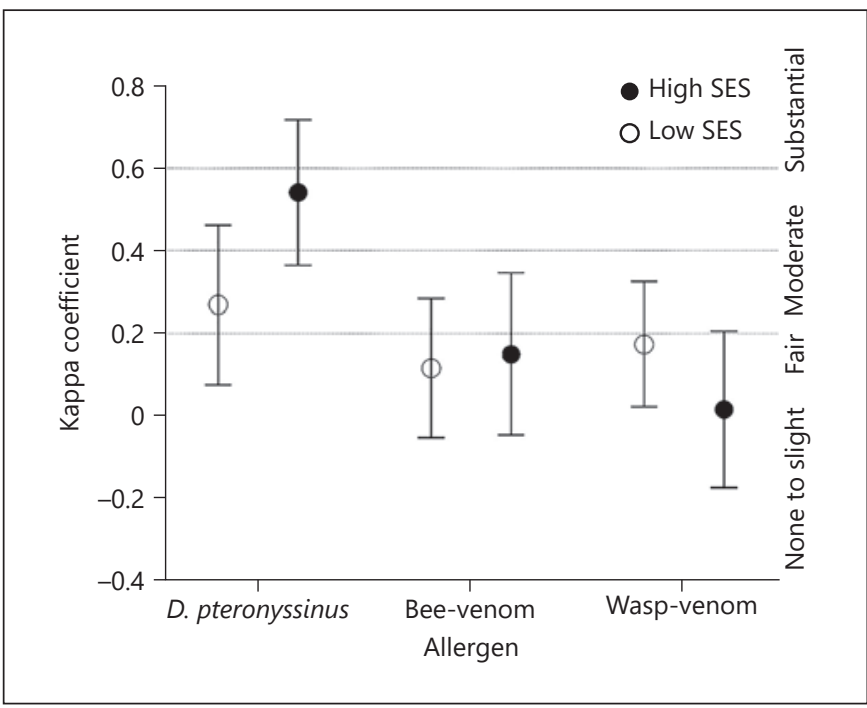

Fig. 2. Agreement between SPT and sIgE to D. pteronyssinus, bee- and wasp-venom in high- and low-SES schoolchildren. Kappa coefficient: $\leq 0.20$ (none to slight agreement); $0.21-0.40$ (fair agreement); 0.41-0.60 (moderate agreement); and 0.610.80 (substantial agreement). SPT, skin prick test; SES, socioeconomic status; bee-venom, Apis mellifera; wasp-venom, Vespula spp.

and Kappa $=0.047$ [ -0.092 to 0.186$], p=0.499$, Fig. 1c, respectively), while a "moderate" agreement (Kappa = 0.434 [0.317-0.550]; $p$ value $<0.001$, Fig. 1a) was found for sensitization to $D$. pteronysssinus

Analyzing the correlation between the wheal size and sIgE levels revealed a correlation for sensitization to $D$. pteronysssinus (rho $=0.431$, $p$ value $<0.001$, Fig. 1a) but neither to bee- nor to wasp-venom (rho: $0.055 ; p$ value $=$ 0.440 , Fig. 1 b; and rho: $0.071 p$ value $=0.321$, Fig. $1 c$, respectively). When SES was considered, no difference was found on the agreement analysis for sensitization to $\mathrm{Hy}$ menoptera venom between high and low SES. We also found no differences on the agreement analysis between helminth-infected versus helminth-uninfected neither between protozoa-infected and protozoa-uninfected as shown in online suppl. Figure 3.

Regarding D. pteronyssinus sensitization, the kappa agreement was stronger in the high than low SES ("moderate," 0.541 [0.364-0.717] vs. "fair," 0.269 [0.076-0.461] for high and low SES, respectively) (Fig. 2). Similarly, when we stratify the population into helminth infected and uninfected, as shown in online suppl. Figure 3, weaker agreement also observed in the helminth-infected (fair agreement: 0.228 [-0.041 to 0.497] compared to helminth-uninfected group (moderate agreement: 0.550 
[0.383-0.716]). However, no differences in the agreement analysis between protozoa infected versus uninfected groups were found.

\section{Discussion}

To the best of our knowledge, this is the first report of a study into the prevalence of Hymenoptera venom sensitization in schoolchildren living in an urban area of a tropical country. Studies on the Hymenoptera venom sensitization so far were performed in temperate or subtropical areas, where exposure to bee and wasp stings is frequent $[3,9]$, and these studies involved a high-risk population such as beekeepers and their relatives [22].

Here, we found the prevalence of skin reactivity to beeand wasp-venom was 14.3 and $12.7 \%$, respectively. A cross-sectional study in Italy reported that 2.98 and $1.45 \%$ of primary schoolchildren were positive for skin test reactivity [3]. It should be noted that the venom concentration used in the Italian study was 3 times lower than in our study. In addition, the test material was purchased from ALK-Abello, while the Italian study used material from Lofarma, which might also contribute to the differences in the prevalence of skin reactivity in the 2 studies. When sIgE to venom was considered, we found the prevalence of positive sIgE to bee- and wasp-venom were 26.5 and $28.5 \%$, respectively, which are similar to the findings in an adult German cohort that reported 23.1 and $31.7 \%$ IgE positivity to bee- and wasp-venom [23].

In our study, majority (88.7\%) of children reported no history of having been stung. From 17 children (11.3\%) who had a history of a sting, only one child (5.9\%) reported LLR but none SSR. The child who reported LLR had a positive skin test, which could suggest a recent exposure to a sting. Proportion of loss of sensitization to Hymenoptera venom in skin tests has been reported to be $12 \%$ per year [24]. The prevalence of clinical reaction in our population is much lower than a questionnaire-based survey in Turkish children [9], which reported 24.3 and 9.9\% for LLR and SSR, respectively. The prevalence of LLR in our study is comparable to a study in Irish children that reported LLR in 5.8\% of the participants [7].

Studies in temperate climates have reported that asymptomatic sensitization to bee- and wasp-venom is a common phenomenon in the general population $[25,26]$. Therefore, it was concluded that detected sensitization to both venoms is clinically irrelevant as the presence of sensitization to both venoms did not translate into clinical reactions. In our study, all children with positive sIgE to either bee- or wasp-venom reported no LLR or SR after being stung. This finding is higher than $69.3 \%$ reported in Denmark [5].

We observed considerable skin reactivity in the absence of sIgE to bee- and wasp venom. This might suggest that skin reactivity to Hymenoptera venom is not necessarily through IgE but through IgE-independent mechanisms [27, 28]. Bee and wasp [29] venom may contain several substances that could provoke toxic reactions [30] by inducing mast cell degranulation directly resulting in positive skin reaction in some children. The low-molecular-weight components of venoms often mediate local toxic and nonallergic reactions while components such as hyaluronidases, phospholipase A1 and A2, antigen 5 , serine proteases, and acid phosphatases do so through $\operatorname{IgE}$ [31].

The absence of skin reactivity despite the presence of sIgE in our population may suggest the presence of IgE with poor biologic activity, which has been described for cross-reactive IgE [32]. The cross-reactive IgE might arise through recognition of Hymenoptera venom components, such as hyaluronidases $[4,33]$. Interestingly, a 44$\mathrm{kD}$ protein similar to hyaluronidase has been shown in mosquito extracts $[34,35]$ and might be responsible for the high sIgE that we observe to venoms. This is in line with our finding of higher sIgE positivity in the low SES, which lives in conditions, associated with inadequate sewage and poor sanitation and, therefore, increased population of mosquitos. There is also a possibility that higher total IgE might contribute to the overall elevation of sIgE as reported by Dold et al. [36].

Similar to our study, the lack of association between sIgE and skin sensitization to venoms has also been reported in a previous study [37]. However, the study was on selected subjects with SR to insect sting which reported that $32 \%$ of participants had negative skin test response. Interestingly, among these subjects, $43 \%$ had venom sIgE antibodies. Discordance between RAST and skin test in their study may reflect the different sensitivity of the 2 tests for diagnosing the venom reactions and it could also be due to differences in the material used in the 2 tests [37].

The asymptomatic sensitization is not only seen to venom allergens, but also to other allergens as already reported in plethora of studies conducted in developing countries [38-40]. In our study, most of the children with detected sIgE or positive skin reactivity to $D$. pteronyssinus did not report clinical symptoms. When stratified according to SES, we observed discordant results in D. pter- 
onyssinus sensitization where the correlation and agreement between SPT and SIgE were weaker in low SES than the high-SES children in particular when considering the proportion of SPT negative among sIgE positive children. Skin sensitization to aeroallergens including HDMs was higher in the high SES than in the low SES, however, no differences were observed in the prevalence of sIgE to $D$. pteronyssinus between high- and low-SES group. This finding was similar to our previous study in the same urban area [11]. In a previous study, thin children have been shown to have less skin sensitivity [41], and however, this is not the case in our study as the zBMI was similar between those positive and negative for SPT to any allergen tested in the low-SES group. In line with our study, KellerFranco et al. [42] showed that nutritional status which was measured by BMI did not affect the skin reactivity to either histamine or D. pteronyssinus.

Low SES has been reported to be associated with lower prevalence of skin reactivity to HDM despite the presence of $\operatorname{sIgE}[40]$. This we have suggested to be due to the down-modulation of skin reactions to allergens by induction of IL-10 [43]. Parasitic infections, in particular, helminths are often highly prevalent in the less affluent populations, leading to expansion of regulatory T cells [44]. However, even though the SPT to venom was lower in low SES schoolchildren, when we tested the agreement between SPT and sIgE, we observed "poor" agreement in both low- and high-SES groups.

One of the limitations of our study is its cross-sectional design which does not allow us to determine causality and time of exposure. It is known that sensitization to venom peaks few weeks after a sting and recedes over time $[24,45]$. In this study, we studied past reactions to venom and current sensitization instead of following up sensitization and reactions after being stung. Moreover, the questionnaire-based approach data on allergy and stingrelated reaction is restricted by recall bias. In addition, the lack of component resolved diagnostic methods in this study may have hampered the evaluation of true sensitization to venom allergy.

Our findings, however, provide evidence that sensitization against bee- or wasp venom is quite prevalent in Indonesian schoolchildren living in an urban area of a tropical region. Higher prevalence of skin reactivity was observed in high-SES than the low-SES children; in contrast, sIgE-positive was more prevalent in the low SES than the high SES. These sensitizations to Hymenoptera venom appear to have poor clinical relevance as they rarely translated into clinical symptoms. Moreover, we also observed discordance between SPT and SIgE to bee- and wasp venom, in particular the high proportions of positive SPT in absence of sIgE, which suggests the direct (non-IgE) effect of venoms resulting in skin reactivity. However, further studies are needed to determine the possible mechanisms underlying this which could be through component resolved diagnostic methods.

\section{Acknowledgements}

The authors would like to thank all participants involved in this study as well as their parents, teachers, and headmasters and we also would like to thank our "IPHIC" field team for their tremendous efforts in sample collection. The authors would like to thank HUM-RC for providing laboratory facilities in the field, and local government and Hasanuddin University for the support. We thank the Joint Scholarship of Directorate General of Resources for Science Technology and Higher Education (DGRSTHE) of Indonesia and Leiden University for providing $\mathrm{PhD}$ scholarship to A.A.

\section{Statement of Ethics}

This study was approved by Health Research Ethics Committee of Faculty of Medicine, Hasanuddin University (Ref: 1504/ H04.8.4.5.31/PP36-KOMETIK/2016). Written informed consent was obtained from all parents prior to data collection.

\section{Conflict of Interest Statement}

The authors have no conflicts of interest to declare.

\section{Funding Sources}

This work was supported by The Ministry of Research and Technology/National Research and Innovation Agency of The Republic of Indonesia (Kemenristek/BRIN) (Grants number: Ref: 7/ AMD/E1/KP. PTNBH/2020 to F.H.) and Leiden University Medical Center, The Netherlands. The sponsor of the study had no role in study design, data collection, data analysis, data interpretation, or writing of the report.

\section{Author Contributions}

Conception and design of the experiments: R.R., M.Y., F.H., and E.S. Data collection and experimentation: A.I.A., J.P.R.K., M.M., S.A.V., S.W., F.H., and E.S. Interpretation of data and statistical analysis: A.I.A.and J.P.R.K. Writing of this paper: A.I.A., J.P.R.K., M.M., S.A.V., S.W., F.H., E.S., R.R., and M.Y. The corresponding author had full access to all of the data in this study and takes final responsibility for the decision to submit it for publication. 


\section{References}

1 Bjornsson E, Janson C, Plaschke P, Norrman E, Sjoberg O. Venom allergy in adult Swedes: a population study. Allergy. 1995 Oct;50(10): 800-5.

2 Schafer T, Przybilla B. IgE antibodies to hymenoptera venoms in the serum are common in the general population and are related to indications of atopy. Allergy. 1996 Jun;51(6): 372-7.

3 Novembre E, Cianferoni A, Bernardini R, Veltroni M, Ingargiola A, Lombardi E, et al. Epidemiology of insect venom sensitivity in children and its correlation to clinical and atopic features. Clin Exp Allergy. 1998 Jul; 28(7):834-8.

4 Blank S, Seismann H, Bockisch B, Braren I, Cifuentes L, McIntyre M, et al. Identification, recombinant expression, and characterization of the $100 \mathrm{kDa}$ high molecular weight Hymenoptera venom allergens Api m 5 and Ves v 3. J Immunol. 2010 May 1;184(9):540313

5 Mosbech H, Tang L, Linneberg A. Insect sting reactions and specific IgE to venom and major allergens in a general population. Int Arch Allergy Immunol. 2016;170(3):194-200.

6 Graif Y, Romano-Zelekha O, Livne I, Green MS, Shohat T. Allergic reactions to insect stings: results from a national survey of 10,000 junior high school children in Israel. J Allergy Clin Immunol. 2006 Jun;117(6):1435-9.

7 Jennings A, Duggan E, Perry IJ, Hourihane JO. Epidemiology of allergic reactions to hymenoptera stings in Irish school children. Pediatr Allergy Immunol. 2010 Dec;21(8):1166-70

8 Gelincik A, İşsever H, Unal D, Işık E, Demirturk M, Gül H, et al. The prevalence of Hymenoptera venom allergy in adults: the results of a very crowded city in Euroasia. Allergol Int. 2015 Jan;64(1):35-40.

9 Arikan-Ayyildiz Z, Isik S, Babus S, Ucku R, Caglayan-Sozmen S, Karaman O, et al. Allergic reactions to Hymenoptera stings in Turkish school children. Allergol Immunopathol. 2016 Jan-Feb;44(1):41-5.

10 Pali-Scholl I, Blank S, Verhoeckx K, Mueller RS, Janda J, Marti E, et al. EAACI position paper: comparing insect hypersensitivity induced by bite, sting, inhalation or ingestion in human beings and animals. Allergy. 2019 May;74(5):874-87.

11 Hamid F, Wahyuni S, van Leeuwen A, van Ree R, Yazdanbakhsh M, Sartono E. Allergic disorders and socio-economic status: a study of schoolchildren in an urban area of Makassar, Indonesia. Clin Exp Allergy. 2015 Jul; 45(7):1226-36.

12 Hamid F, Wiria AE, Wammes LJ, Kaisar MM, Djuardi Y, Versteeg SA, et al. Risk factors associated with the development of atopic sensitization in Indonesia. PLoS One. 2013;8(6): e67064.

13 Lange J, Cichocka-Jarosz E, Marczak H, Krauze A, Tarczoń I, Świebocka E, et al. Natural history of Hymenoptera venom allergy in children not treated with immunotherapy. Ann Allergy Asthma Immunol. 2016 Mar; 116(3):225-9

14 Bilo BM, Rueff F, Mosbech H, Bonifazi F, Oude-Elberink JN. Diagnosis of Hymenoptera venom allergy. Allergy. 2005 Nov;60(11): 1339-49.

15 Heinzerling L, Mari A, Bergmann KC, Bresciani M, Burbach G, Darsow U, et al. The skin prick test: European standards. Clin Transl Allergy. 2013 Feb 1;3(1):3.

16 Hamid F, Wiria AE, Wammes LJ, Kaisar MM, Lell B, Ariawan I, et al. A longitudinal study of allergy and intestinal helminth infections in semi urban and rural areas of Flores, Indonesia (ImmunoSPIN Study). BMC Infect Dis. 2011 Apr 1;11:83.

17 Verweij JJ, Canales M, Polman K, Ziem J, Brienen EA, Polderman AM, et al. Molecular diagnosis of Strongyloides stercoralis in faecal samples using real-time PCR. Trans R Soc Trop Med Hyg. 2009 Apr;103(4):342-6.

18 Verweij JJ, Blangé RA, Templeton K, Schinkel J, Brienen EA, van Rooyen MA, et al. Simultaneous detection of Entamoeba histolytica, Giardia lamblia, and Cryptosporidium parvum in fecal samples by using multiplex realtime PCR. J Clin Microbiol. 2004 Mar;42(3): 1220-3.

19 Verweij JJ, Mulder B, Poell B, van Middelkoop D, Brienen EA, van Lieshout L. Realtime PCR for the detection of Dientamoeba fragilis in fecal samples. Mol Cell Probes. 2007 Oct-Dec;21(5-6):400-4.

20 Amaruddin AI, Hamid F, Koopman JPR, Muhammad M, Brienen EA, van Lieshout L, et al. The bacterial gut microbiota of schoolchildren from high and low socioeconomic status: a study in an Urban Area of Makassar, Indonesia. Microorganisms. 2020 Jun 26;8(6).

21 Landis JR, Koch GG. The measurement of observer agreement for categorical data. Biometrics. 1977 Mar;33(1):159-74.

22 Muller UR. Bee venom allergy in beekeepers and their family members. Curr Opin Allergy Clin Immunol. 2005 Aug;5(4):343-7.

23 Blank S, Haemmerle S, Jaeger T, Russkamp D, Ring J, Schmidt-Weber CB, et al. Prevalence of Hymenoptera venom allergy and sensitization in the population-representative German KORA cohort. Allergo J Int. 2019 Sep 1; 28(6):183-91.

24 Golden DB, Marsh DG, Freidhoff LR, Kwiterovich KA, Addison B, Kagey-Sobotka A, et al. Natural history of Hymenoptera venom sensitivity in adults. J Allergy Clin Immunol. 1997 Dec;100(6 Pt 1):760-6.

25 Sturm GJ, Schuster C, Kranzelbinder B, Wiednig M, Groselj-Strele A, Aberer W. Asymptomatic sensitization to hymenoptera venom is related to total immunoglobulin $\mathrm{E}$ levels. Int Arch Allergy Immunol. 2009; 148(3):261-4.

26 Sturm GJ, Kranzelbinder B, Schuster C, Sturm EM, Bokanovic D, Vollmann J, et al.
Sensitization to Hymenoptera venoms is common, but systemic sting reactions are rare. J Allergy Clin Immunol. 2014 Jun; 133(6):1635-e1.

27 Galli SJ, Starkl P, Marichal T, Tsai M. Mast cells and IgE in defense against venoms: possible "good side" of allergy? Allergol Int. 2016 Jan;65(1):3-15.

28 Galli SJ, Starkl P, Marichal T, Tsai M. Mast cells and IgE can enhance survival during innate and acquired host responses to venoms. Trans Am Clin Climatol Assoc. 2017;128: 193-221.

29 Hirai Y, Yasuhara T, Yoshida H, Nakajima T, Fujino M, Kitada C. A new mast cell degranulating peptide "mastoparan" in the venom of Vespula lewisii. Chem Pharm Bull. 1979 Aug; 27(8):1942-4.

30 Wright DN, Lockey RF. Local reactions to stinging insects (Hymenoptera). Allergy Proc. 1990 Jan-Feb;11(1):23-8.confproc

31 Palm NW, Rosenstein RK, Yu S, Schenten DD, Florsheim E, Medzhitov R. Bee venom phospholipase A2 induces a primary type 2 response that is dependent on the receptor ST2 and confers protective immunity. Immunity. 2013 Nov 14;39(5):976-85.

32 van der Veen MJ, van Ree R, Aalberse RC, Akkerdaas J, Koppelman SJ, Jansen HM, et al. Poor biologic activity of cross-reactive IgE directed to carbohydrate determinants of glycoproteins. J Allergy Clin Immunol. 1997 Sep; 100(3):327-34.

33 Eberlein B, Krischan L, Darsow U, Ollert M, Ring J. Double positivity to bee and wasp venom: improved diagnostic procedure by recombinant allergen-based IgE testing and basophil activation test including data about cross-reactive carbohydrate determinants. J Allergy Clin Immunol. 2012 Jul;130(1):155-61.

34 Sabbah A, Hassoun S, Drouet M, Lauret MG, Doucet M. [The wasp/mosquito syndrome]. Allerg Immunol. 1999 Jun;31(6):175-84.

35 Scala E, Pirrotta L, Uasuf CG, Mistrello G, Amato S, Guerra EC, et al. Aedes communis reactivity is associated with bee venom hypersensitivity: an in vitro and in vivo study. Int Arch Allergy Immunol. 2018;176(2):1015.

36 Dold S, Heinrich J, Wichmann HE, Wjst M. Ascaris-specific IgE and allergic sensitization in a cohort of school children in the former East Germany. J Allergy Clin Immunol. 1998 Sep;102(3):414-20.

37 Golden DB, Kagey-Sobotka A, Norman PS, Hamilton RG, Lichtenstein LM. Insect sting allergy with negative venom skin test responses. J Allergy Clin Immunol. 2001 May; 107(5): 897-901.

38 Vereecken K, Kanobana K, Wördemann M, Junco Diaz R, Menocal Heredia L, Ruiz Espinosa A, et al. Associations between atopic markers in asthma and intestinal helminth infections in Cuban schoolchildren. Pediatr Allergy Immunol. 2012 Jun;23(4):332-8 
39 Obeng BB, Amoah AS, Larbi IA, de Souza DK, Uh HW, Fernández-Rivas M, et al. Schistosome infection is negatively associated with mite atopy, but not wheeze and asthma in Ghanaian schoolchildren. Clin Exp Allergy. 2014 Jul;44(7):965-75.

40 Alcantara-Neves NM, Veiga RV, Ponte JC, da Cunha SS, Simões SM, Cruz ÁA, et al. Dissociation between skin test reactivity and antiaeroallergen IgE: determinants among urban Brazilian children. PLoS One. 2017;12(3): e0174089.
41 Calvert J, Burney P. Effect of body mass on exercise-induced bronchospasm and atopy in African children. J Allergy Clin Immunol. 2005 Oct;116(4):773-9.

42 Keller Franco A, Oselka Sarni R, Carvalho Mallozi M, Solé D. Body mass index and skin reactivity to histamine and Dermatophagoides pteronyssinus in children and adolescents followed in a pediatric allergy service. Eur Ann Allergy Clin Immunol. 2017 May;49(3): 110-3.
43 van den Biggelaar AH, van Ree R, Rodrigues LC, Lell B, Deelder AM, Kremsner PG, et al. Decreased atopy in children infected with Schistosoma haematobium: a role for parasite-induced interleukin-10. Lancet. 2000 Nov 18;356(9243):1723-7.

44 Smits HH, Everts B, Hartgers FC, Yazdanbakhsh M. Chronic helminth infections protect against allergic diseases by active regulatory processes. Curr Allergy Asthma Rep. 2010 Jan; 10(1):3-12.

45 Stoevesandt J, Hosp C, Kerstan A, Trautmann A. Sensitization to Hymenoptera venom marker allergens: prevalence, predisposing factors, and clinical implications. Clin Exp Allergy. 2018 Dec;48(12):1735-43. 